\title{
QUANTITATIVE MULTIPLE POINTWISE CONVERGENCE AND EFFECTIVE MULTIPLE CORRELATIONS
}

\author{
RENE RÜHR AND RONGGANG SHI
}

\begin{abstract}
We show that effective $2 \ell$-multiple correlations imply quantitative $\ell$-multiple pointwise ergodic theorems. The result has a wide class of applications which include subgroup actions on homogeneous spaces, ergodic nilmanifold automorphisms, subshifts of finite type and Young towers.
\end{abstract}

\section{INTRODUCTION}

Let $h: X \rightarrow X$ be a measure preserving map on a probability space $(X, \mathcal{X}, \mu)$. The $\ell$-multiple pointwise ergodic theorem asks about the almost sure convergence of the average

$$
\frac{1}{N} \sum_{n=1}^{N} f_{1}\left(h^{n} x\right) f_{2}\left(h^{2 n} x\right) \cdots f_{\ell}\left(h^{\ell n} x\right) \quad(N \rightarrow \infty),
$$

where $f_{i}: X \rightarrow \mathbb{R}(1 \leqslant i \leqslant \ell)$ are bounded measurable functions. When $\ell=1$, the almost sure convergence of (1.1) is the Birkhoff ergodic theorem. The study of the question for $\ell=2$ is initiated by Hillel Furstenberg [12, p. 96]. Furstenberg's question was answered affirmatively by Bourgain [6].

Derrien and Lesigne [8] showed that (1.1) converges almost surely for all $f_{i} \in L^{\infty}(X, \mathcal{X}, \mu)$ if and only if the same holds for all $f_{i} \in L^{\infty}(X, \mathcal{P}, \mu)$ where $\mathcal{P}$ is the Pinsker $\sigma$-algebra of $h$. In this paper all the function spaces such as $L^{p}, C_{c}^{\infty}$ and $C^{\theta}$ consist of real valued functions. Therefore, the almost sure convergence of (1.1) holds if $h$ is a Kolmogorov automorphism. Since every Bernoulli automorphism is a Kolmogorov automorphism, the same result holds if $h$ is a two-sided Bernoulli shift. The same method was used by Assani [1] to show the almost sure convergence of (1.1) when $h$ is weakly mixing and the restriction of $h$ to $(X, \mathcal{P}, \mu)$ has singular spectrum. We refer the readers to Walters [25] for the definitions of these concepts.

Recently, progress on higher order pointwise convergence was obtained by Huang-Shao-Ye [16] for ergodic distal systems and by Gutman-Huang-ShaoYe [15] for weakly mixing pairwise independent systems. See also Donoso-Sun [10] [11] for an extension of [16] to commuting distal transformations.

2010 Mathematics Subject Classification. Primary 37A30; Secondary 37A25, 37C85.

Key words and phrases. pointwise convergence, multiple mixing, ergodic theorem.

R.R. acknowledges that this project has received funding from the European Research Council (ERC) under the European Union's Horizon 2020 research and innovation program (grant agreement No. 754475).

R.S. is supported by NSFC 11871158 . 
We remark here that the multiple mean ergodic theorem for commuting maps are well-understood, see Tao [24] and references there. An interesting extension of the mean ergodic theorem to nilpotent group actions with polynomial ergodic averages was obtained by Walsh [26]. The pointwise convergence of polynomial ergodic averages is known for $\ell=1$ by Bourgain [4] [5] and for Kolmogorov automorphisms by [8].

In a recent paper [22, the authors noticed that effective multiple correlations can be used to prove quantitative multiple pointwise convergence. The aim of this paper is to develop this method further in an abstract setting (Theorem 2.2) and obtain more results on quantitative multiple pointwise convergence. The examples contain commuting subgroup actions on homogeneous spaces, nilmanifold automorphisms, subshifts of finite type and Young towers.

We begin with subgroup actions on homogeneous spaces. A unitary representation of a locally compact topological group $H$ on a (real) Hilbert space $V$ is said to have a spectral gap if there exists $\delta>0$ and a compactly supported probability measure $\nu$ on $H$ such that

$$
\left\|\int_{H} h v \mathrm{~d} \nu(h)\right\| \leqslant(1-\delta)\|v\| \quad \text { for all } v \in V .
$$

Let $\Gamma$ be a lattice of a connected Lie group $G$. The homogeneous space $X=G / \Gamma$ carries a unique $G$ invariant probability measure $\mu$. Let $H$ be a connected semi-simple subgroup of $G$ with finite center. The natural left translation action of $H$ on $X$ induces a unitary representation of $H$ on

$$
L_{0}^{2}(X, \mu)=\left\{f \in L^{2}(X, \mu): \mu(f)=0\right\} \quad \text { via } \quad(h f)(x)=f\left(h^{-1} x\right) .
$$

We say the action of $H$ on $X$ has a strong spectral gap if the representation of each noncompact simple factor of $H$ on $L_{0}^{2}(X, \mu)$ has a spectral gap. A sequence of integers $\left\{r_{n}\right\}$ is said to be non-clustered if the cardinality of $\{n \in$ $\left.\mathbb{N}: r_{n}=m\right\}$ is uniformly bounded for all $m \in \mathbb{Z}$. In this paper we adopt the convention that the set of natural numbers $\mathbb{N}$ does not contain 0 . For $h \in H$, we let $\langle h\rangle$ be the group generated by $h$. We use $C_{c}^{\infty}(X)$ to denote the space of compactly supported and smooth functions on $X$. We call a group element $h \in H$ quasi-unipotent if all the eigenvalues of its corresponding adjoint action $\operatorname{Ad}(h) \in \operatorname{GL}(\operatorname{Lie}(H))$ have modulus one.

Theorem 1.1. Let $G, \Gamma, X, \mu, H$ be as above. Suppose the action of $H$ on $X$ has a strong spectral gap. Let $h_{1}, \ldots, h_{\ell}$ be commuting elements of $H$ such that all the groups $\left\langle h_{i}\right\rangle$ and $\left\langle h_{i} h_{j}^{-1}\right\rangle(i \neq j)$ are unbounded. Then there exists $\varepsilon>0$ such that for any $\ell \in \mathbb{N}$, any $f_{1}, \ldots, f_{\ell} \in C_{c}^{\infty}(X)$ and any non-clustered sequence of integers $\left\{r_{n}\right\}$, one has $\mu$-almost surely

$$
\frac{1}{N} \sum_{n=1}^{N} f_{1}\left(h_{1}^{r_{n}} x\right) \cdots f_{\ell}\left(h_{\ell}^{r_{n}} x\right)=\mu\left(f_{1}\right) \cdots \mu\left(f_{\ell}\right)+o_{x}\left(N^{-\varepsilon}\right) .
$$


Moreover, if all the $h_{i}$ and $h_{i} h_{j}^{-1}(i \neq j)$ are not quasi-unipotent, then (1.2) holds almost surely with a better error term

$$
o_{x}\left(N^{-\frac{1}{2}} \log ^{\frac{3}{2}+\varepsilon} N\right) \quad \forall \varepsilon>0 .
$$

Here the notation $\varphi(N)=o(\psi(N))$ means $\lim _{N \rightarrow \infty} \frac{\varphi(N)}{\psi(N)}=0$. In this paper the phrase quantitative is used to denote a weaker version of effectiveness in the sense that we do not have any control of the convergence speed in the little $o$ notation appearing in the ergodic theorems. Theorem 1.1 is already interesting in the simplest case where $\ell=1$ as can be seen from the following example.

Example 1.2. Theorem 1.1 holds for $H=G=\mathrm{SL}_{2}(\mathbb{R}), \Gamma=\mathrm{SL}_{2}(\mathbb{Z}), \ell=1$, $h_{1}=u(s)=\left(\begin{array}{ll}1 & s \\ 0 & 1\end{array}\right)$ where $s \neq 0$ and $r_{n}=p(n)$ where $p(n)$ is a polynomial with integer coefficients and degree at least two. The qualitative result in this case is known earlier by [4]. According to a conjecture of Shah [21], it is expected that the qualitative result holds for all the generic $x$, where $x$ is said to be generic if the trajectory $\{u(t) x: t \geqslant 0\}$ is dense in $X$.

Another interesting case is to take $r_{n}$ to be the $n$-th prime number. This type of questions was considered earlier by Sarnak and Ubis [19]. It is also expected that the qualitative result holds for all the generic $x$.

Next we consider the multiple ergodic theorem for a fixed probability measure preserving system $(X, \mathcal{X}, \mu, h)$. Usually when $X$ is a topological space, we take $\mathcal{X}$ to be the Borel $\sigma$-algebra. In this case or other cases where $\mathcal{X}$ is understood or does not play an important role, we may omit it in our notation. We say $(X, \mu, h)$ is $k$-mixing with rate $n^{-\delta}(\delta>0)$ for a vector space $\mathcal{F}$ of measurable functions on $X$ if for any $f_{0}, f_{1}, \ldots, f_{k} \in \mathcal{F}$ and any pairwise distinct $n_{0}, n_{1}, \ldots, n_{k} \in \mathbb{N}$, one has

$$
\int_{X} f_{0}\left(h^{n_{0}} x\right) \cdots f_{k}\left(h^{n_{k}} x\right) \mathrm{d} \mu=\mu\left(f_{0}\right) \cdots \mu\left(f_{k}\right)+O\left(\inf _{i \neq j}\left|n_{i}-n_{j}\right|^{-\delta}\right),
$$

where the implied constant is allowed to depend on $k$ and $f_{1}, \ldots, f_{k}$. Here $\varphi=O(\psi)$ for two functions means $|\varphi| \leqslant C|\psi|$ for some constant $C>0$. Our definition of $k$-mixing follows the convention of Glasner [13, Chapter 3 §9]. The pairwise distinctness assumption for $n_{1}, \ldots, n_{k} \in \mathbb{N}$ is not essential, since we could drop it and and replace $\left|n_{i}-n_{j}\right|^{-\delta}$ by $\left(\left|n_{i}-n_{j}\right|+1\right)^{-\delta}$.

Theorem 1.3. If a probability measure preserving system $(X, \mathcal{X}, \mu, h)$ is $(2 \ell-$ $1)$-mixing with rate $n^{-\delta}(\delta>0)$ for a vector space of measurable functions $\mathcal{F}$ containing constant functions, then for any pairwise distinct $m_{1}, \ldots, m_{\ell} \in \mathbb{N}$, any $f_{1}, \ldots, f_{\ell} \in \mathcal{F}$ and any non-clustered sequence of positive integers $\left\{r_{n}\right\}$, one has $\mu$-almost surely

$$
\frac{1}{N} \sum_{n=1}^{N} f_{1}\left(h^{m_{1} r_{n}} x\right) \cdots f_{\ell}\left(h^{m_{\ell} r_{n}} x\right)=\mu\left(f_{1}\right) \cdots \mu\left(f_{\ell}\right)+o_{x}\left(\rho_{\varepsilon, \delta}(N)\right),
$$


where $\rho_{\varepsilon, \delta}(N)=N^{-\frac{1}{2}} \log ^{\frac{3}{2}+\varepsilon} N$ if $\delta>1$ and $\rho_{\varepsilon, \delta}(N)=N^{-\frac{\delta}{2}+\varepsilon}$ if $0<\delta \leqslant 1$.

Remark 1.4. We say $(X, \mu, h)$ is $k$-mixing with exponential rate if there exists $\sigma>0$ such that the error term of (1.4) is given by $O\left(e^{-\sigma \inf _{i \neq j}\left|n_{i}-n_{j}\right|}\right)$. Clearly, exponential rate implies $n^{-2}$ rate and hence Theorem 1.3 holds in this case.

Remark 1.5. If we assume $h: X \rightarrow X$ is invertible and (1.4) holds for any $n_{i} \in \mathbb{Z}$ with $k=2 \ell-1$, then (1.5) holds almost surely with pairwise distinct nonzero integers $m_{1}, \cdots, m_{\ell}$.

Example 1.6. Nilmanifold automorphisms Let $\Gamma$ be a lattice of a simply connected nilpotent Lie group $G$. The homogeneous space $X=G / \Gamma$ is called a compact nilmanifold. An automorphism $h$ is a diffeomorphism of $X$ which lifts to an automorphism of $G$. We assume that $h$ is ergodic with respect to the probability Haar measure $\mu$ on $X$.

We endow $G$ with a right invariant Riemannian metric and consider $X$ as a metric space with the induced metric. We fix $\theta>0$ and take $\mathcal{F}=C^{\theta}(X)$ to be the space of $\theta$-Hölder continuous functions on $X$. It follows from [14, Theorem 1.2] that $(X, \mu, h)$ is mixing with exponential rate. In view of Remark [1.4, Theorem 1.3 in the form of Remark 1.5 holds for any $\ell \geqslant 1$.

Example 1.7. Subshifts of finite type Let $h$ be the left translation on the space $\Sigma=\{1, \ldots, m\}^{\mathbb{Z}}$ where $m \geqslant 2$. We endow $\Sigma$ with a metric so that the distance of two points $\mathbf{x}=\left(x_{i}\right)$ and $\mathbf{y}=\left(y_{i}\right)$ are given by $\mathrm{d}(\mathbf{x}, \mathbf{y})=2^{-n}$, where $n$ is the largest nonnegative integer with $x_{i}=y_{i}$ for every $|i|<n$. Let $A$ be an $m \times m$ aperiodic matrix with entries 0 or 1 , i.e., there exists $n \geqslant 1$ such that all the entries of $A^{n}$ are positive. Let

$$
\Sigma_{A}=\left\{\mathbf{x}=\left(x_{i}\right) \in \Sigma: A_{x_{i} x_{i+1}}=1 \quad \forall i \in \mathbb{Z}\right\} .
$$

Then the dynamical system $\left(\Sigma_{A}, h\right)$ is topologically mixing, see [7, Lemma 1.3]. Let $\theta>0$ and $\mathcal{F}=C^{\theta}(X)$ be the space of $\theta$-Hölder continuous functions on $X$. Let $\mu=\mu_{f}$ be the Gibbs measure given by some $f \in C^{\theta}(X)$, i.e., $\mu$ is the unique $h$ invariant probability measure on $X$ maximizing the pressure given by $f$, see [7, Theorem 1.22]. The effective multiple mixing with exponential rate was essentially proved by Kotani-Sunada [18] which uses a different definition of correlations. We will give a proof of effective multiple mixing based on [18] in 33 , In view of Remark 1.4, the conclusion of Theorem 1.3 in the form of Remark 1.5 holds for all $\ell \in \mathbb{N}$.

In particular, let $\nu$ be the probability measure on $\{1, \ldots, m\}$ given by a probability vector $\left(p_{1}, \ldots, p_{m}\right)$, i.e., $\nu(i)=p_{i}>0$. Then the measure $\mu=\nu^{\otimes \mathbb{Z}}$ is a Gibbs measure on $\Sigma$, see [25, Theorem 9.16]. Therefore, Theorem 1.3$]$ implies a quantitative version of multiple ergodic theorem for all the finite Bernoulli shifts.

It is easy to see that if the quantitative multiple convergence holds for a measure preserving system $(X, \mu, h)$, then it holds for its factors. Here we say 
$(Y, \nu, g)$ is a factor of $(X, \mu, h)$, if there is a measurable map $\varphi: X \rightarrow Y$ such that $\varphi_{*} \mu=\nu$ and $\varphi h=g \varphi$. In particular, Theorem 1.3 holds for topologically mixing Anosov diffeomorphisms endowed with Gibbs measures associated to smooth functions, where we take $\mathcal{F}$ to be the space of smooth functions.

In certain non-invertible systems, effective mixing decays polynomially with coefficients given by the $L^{\infty}$ norm of a test function. An important family of dynamical systems having this property are Young towers [27]. In this case effective multiple mixing follows from effective mixing. As a corollary, one gets the quantitative multiple pointwise convergence. This is the content of the next theorem.

Theorem 1.8. Let $(X, \mu, h)$ be a probability measure preserving system. Let $\mathcal{L}$ be a subspace of $L^{2}(X, \mu)$ containing constant functions and let $\|\cdot\|_{\mathcal{L}}$ be a norm on $\mathcal{L}$. Suppose there exist $\delta, C>0$ such that for any $\varphi \in L^{\infty}(X, \mu)$, $\psi \in \mathcal{L}$ and $n \in \mathbb{N}$ we have

$$
\left|\int_{X} \varphi\left(h^{n} x\right) \psi(x) \mathrm{d} \mu(x)-\int_{X} \varphi \mathrm{d} \mu \int_{X} \psi \mathrm{d} \mu\right| \leqslant C\|\varphi\|_{L^{\infty}}\|\psi\|_{\mathcal{L}} n^{-\delta}
$$

Then for any $k \in \mathbb{N}$ the system $(X, \mu, h)$ is $k$-mixing with rate $n^{-\delta}$ for the vector space $\mathcal{L} \cap L^{\infty}(X, \mu)$. Hence Theorem 1.3 holds.

Although the almost sure convergence theorems considered in the introduction are with respect to invariant measures, the general result Theorem 2.2 does not require any invariance of the measure, but only the effective correlations. The multiple pointwise convergence considered in the appendix of [22] is of this type. Several results of effective multiple correlations are obtained by Dolgopyat [9, Theorem 2] for partially hyperbolic systems. The results of this paper (e.g. Theorem 3.1) can be applied to the settings there to get quantitative multiple pointwise convergence.

Acknowledgements: We would like to thank Wen Huang, Hanfeng Li and Weixiao Shen for drawing our attention to [8] [1], [26] and [27] respectively.

\section{General Quantitative multiple COnVERGence}

In this section we prove the general version of quantitative multiple convergence Theorem 2.2. A simplified version is given in Theorem 3.1 under the assumption of effective decay of correlations. The following theorem is the main tool to prove Theorem 2.2 .

Theorem 2.1. Let $(Y, \nu)$ be a probability space and let $F_{k}: Y \rightarrow \mathbb{R}$ be a sequence of square integrable functions. Suppose there exist $C, \sigma>0$ such that for any nonnegative integers $m<n$

$$
\int_{Y}\left(\sum_{m<k \leqslant n} F_{k}(y)\right)^{2} \mathrm{~d} \nu(y) \leqslant C\left(n^{\sigma}-m^{\sigma}\right) .
$$


Then given any $\varepsilon>0$, one has

$$
\lim _{N \rightarrow \infty} \frac{\sum_{1 \leqslant k \leqslant N} F_{k}(y)}{N^{\frac{\sigma}{2}} \log ^{\frac{3}{2}+\varepsilon} N}=0
$$

for $\nu$-almost every $y \in Y$.

If $0<\sigma \leqslant 1$ and $F_{k}(y)=f_{k}(y)-b_{k}$ where $f_{k}$ is nonnegative and $0 \leqslant b_{k} \leqslant$ $k^{\sigma}-(k-1)^{\sigma}$, then Theorem 2.1] is a special case of [23, Chapter 1 Lemma 10]. The proof of the theorem is essentially the same as that in [23] based on dyadic decomposition, and the method is attributed to Schmidt [20]. The extension to the case where $1<\sigma<2$ allows us to prove quantitative ergodic theorems in the case of polynomial decay of correlations. On the other hand, the case where $\sigma \geqslant 2$ does not provide useful information in the application to the usual ergodic theorem, but it might be useful in other contexts.

Proof. For nonnegative integers $r \leqslant s$, let $L_{s, r}$ be the class of sets $\{m \in \mathbb{N}$ : $\left.i 2^{r}<m \leqslant(i+1) 2^{r}\right\},\left(0 \leqslant i<2^{s-r}\right)$, and let $L_{s}=\bigcup_{0 \leqslant r<s} L_{s, r}$. For each positive integer $n<2^{s}$, there exists a subset $L(n)$ of $L_{s}$ with cardinality at most $s$ such that $\{1,2, \ldots, n\}$ is a disjoint union of $I \in L(n)$, see [17, Lemma 3.4]. We take $s(n)$ to be the smallest integer $s$ such that $n<2^{s}$. Then for any $\varepsilon, \sigma>0$

$$
\limsup _{n \rightarrow \infty} \frac{2^{s(n) \sigma / 2} s(n)^{\frac{3}{2}+\varepsilon}}{n^{\sigma / 2} \log ^{\frac{3}{2}+\varepsilon} n}<\infty .
$$

For each positive integer $s$, in view of (2.1) we have

$$
\int_{Y} \sum_{I \in L_{s}}\left(\sum_{k \in I} F_{k}(y)\right)^{2} \mathrm{~d} \nu(y)=\sum_{0 \leqslant r<s} \sum_{I \in L_{s, r}} \int_{Y}\left(\sum_{k \in I} F_{k}(y)\right)^{2} \mathrm{~d} \nu(y) \leqslant C s 2^{\sigma s} .
$$

Let

$$
Y_{s}=\left\{y \in Y: \sum_{I \in L_{s}}\left(\sum_{k \in I} F_{k}(y)\right)^{2}>s^{2+\varepsilon} 2^{\sigma s}\right\} .
$$

By (2.3) and Chebyshev's inequality, we have $\nu\left(Y_{s}\right) \leqslant C s^{-1-\varepsilon}$. Since $\sum_{s=1}^{\infty} \nu\left(Y_{s}\right)<$ $\infty$, the Borel-Cantelli lemma implies that there is a full measure set $Y_{\varepsilon}$ such that every $y \in Y_{\varepsilon}$ does not belong to $Y_{s}$ for $s$ sufficiently large.

If $y \notin Y_{s}$ and $n<2^{s}$, then by Cauchy-Schwarz inequality and (2.4), we have

$$
\begin{aligned}
\left(\sum_{k=1}^{n} F_{k}(y)\right)^{2} & =\left(\sum_{I \in L(n)} \sum_{k \in I} F_{k}(y)\right)^{2} \leqslant s \sum_{I \in L(n)}\left(\sum_{k \in I} F_{k}(y)\right)^{2} \\
& \leqslant s \sum_{I \in L_{s}}\left(\sum_{k \in I} F_{k}(y)\right)^{2} \leqslant s^{3+\varepsilon} 2^{\sigma s} .
\end{aligned}
$$


For $y \in Y_{\varepsilon}$, by (2.2) and (2.5)

$$
\lim _{n \rightarrow \infty} \frac{\left|\sum_{1 \leqslant k \leqslant n} F_{k}(y)\right|}{n^{\frac{\sigma}{2}} \log ^{\frac{3}{2}+\varepsilon} n}=\lim _{n \rightarrow \infty} \frac{\left|\sum_{1 \leqslant k \leqslant n} F_{k}(y)\right|}{2^{s(n) \sigma / 2} s(n)^{\frac{3}{2}+\frac{\varepsilon}{2}}} \frac{1}{s(n)^{\frac{\varepsilon}{2}}} \frac{2^{s(n) \sigma / 2} s(n)^{\frac{3}{2}+\varepsilon}}{n^{\frac{\sigma}{2}} \log ^{\frac{3}{2}+\varepsilon} n}=0 .
$$

Now we apply Theorem 2.1 to the question of multiple pointwise convergence. Let $X, Y$ be measurable spaces and let $\ell$ be a positive integer. For $1 \leqslant i \leqslant \ell$ and $n \in \mathbb{N}$, let $\xi_{i}: Y \rightarrow X, h_{i, n}: X \rightarrow X$ and $f_{i}: X \rightarrow \mathbb{R}$ be measurable maps. The $\ell$-multiple pointwise convergence considers the average of the map $F_{n}: Y \rightarrow \mathbb{R}$ defined by

$$
F_{n}(y)=f_{1}\left(h_{1, n} \xi_{1}(y)\right) f_{2}\left(h_{2, n} \xi_{2}(y)\right) \cdots f_{\ell}\left(h_{\ell, n} \xi_{\ell}(y)\right)=\prod_{i=1}^{\ell} f_{i}\left(h_{i, n} \xi_{i}(y)\right) .
$$

Let $\nu$ be a probability measure on $Y$ such that all the $F_{n}(n \in \mathbb{N})$ are square integrable. We assume that there exists $M, \delta>0$ and a family of functions $b_{i, j}: \mathbb{N} \times \mathbb{N} \rightarrow[1, \infty], c_{i, j}: \mathbb{N} \rightarrow[1, \infty]$ where $i, j \in\{1, \ldots, \ell\}$ such that

$$
\left|\int_{Y} F_{m}(y) F_{n}(y) \mathrm{d} \nu(y)\right| \leqslant M \sum_{i, j=1}^{\ell}\left(b_{i, j}(m, n)^{-\delta}+c_{i, j}(m)^{-\delta}+c_{i, j}(n)^{-\delta}\right)
$$

for all $m, n \in \mathbb{N}$. We make it convention that $\infty^{-\delta}=0$, and hence $b_{i, j}(m, n)=$ $\infty$ means this term does not contribute to the upper bound estimate. We should interpret (2.6) as effective $2 \ell$-multiple correlations whose error term is given by each pair of functions or a single term. The simplified setting in $\$ 3$ and (3.3) may help the reader understand the assumption (2.6). We denote the cardinality of a subset $S$ of $\mathbb{N}$ by $|S|$.

Theorem 2.2. Let the notation and assumptions be as above. We assume that for all $1 \leqslant i, j \leqslant \ell$ and $n \in \mathbb{N}$

$$
\left|\left\{k \in \mathbb{N}: c_{i, j}(k) \leqslant n\right\}\right| \leqslant M n .
$$

For any $1 \leqslant i, j \leqslant \ell$, we assume either for all $m, n \in \mathbb{N}$

$$
\left|\left\{k \in \mathbb{N}: b_{i, j}(m, k) \leqslant n\right\}\right| \leqslant M n,
$$

or for all $m, n \in \mathbb{N}$

$$
\left|\left\{k \in \mathbb{N}: b_{i, j}(k, m) \leqslant n\right\}\right| \leqslant M n .
$$

Then given any $\varepsilon>0$, for $\nu$-almost every $y \in Y$ one has

$$
\frac{1}{N} \sum_{k=1}^{N} F_{k}(y)=o_{y}\left(\rho_{\varepsilon, \delta}(N)\right)
$$

where $\rho_{\varepsilon, \delta}(N)$ is as in Theorem 1.3. 
We use the same constant $M$ in (2.7), (2.8) and (2.9) for the sake of explicitness, and in applications it suffices to check that the left hand sides of them are « $n$ with the implied constant independent of $m$ or $n$. Here and hereafter the notation $\varphi \ll \psi$ for two nonnegative functions means $\varphi \leqslant C \psi$ for a constant $C>0$. It is not hard to see that (2.7) holds if and only if we can rearrange the sequence $\left\{c_{i, j}(n)\right\}$ increasingly so that the integral part of the $k$ th term is larger than or equal to $\frac{k}{M}$. Here the integral part of a real number $s$ is the least integer bigger than or equal to $s$. We shall make use of this fact in the following way: given any subset $\left\{n_{1}, \ldots, n_{\ell}\right\} \subset \mathbb{N}$, ordered in such a way that $c_{i, j}\left(n_{k}\right)$ is increasing for $\ell=1, \ldots, k$, then $c_{i, j}\left(n_{k}\right)^{-1} \leqslant \frac{2 M}{k}$.

We also note that there is the following sufficient condition: (2.7) holds if for any $s \in \mathbb{N}$

$$
\left|\left\{k \geqslant 1: c_{i, j}(k) \in[s, s+1]\right\}\right| \leqslant M .
$$

Similarly, (2.8) holds if

$$
\left|\left\{k \geqslant 1: b_{i, j}(m, k) \in[s, s+1]\right\}\right| \leqslant M
$$

for all $m \in \mathbb{N}$. A similar sufficient condition can be given for (2.9).

Proof of Theorem 2.1. By (2.6), for any nonnegative integers $m<n$ we have

$$
\begin{aligned}
E(m, n) & :=\int_{Y}\left(\sum_{m<k \leqslant n} F_{k}(y)\right)^{2} \mathrm{~d} \nu(y) \\
& \leqslant \sum_{m<s, t \leqslant n}\left|\int_{Y} F_{s} F_{t} \mathrm{~d} \nu(y)\right| \\
& \leqslant M \sum_{m<s, t \leqslant n} \sum_{1 \leqslant i, j \leqslant \ell}\left(b_{i, j}(s, t)^{-\delta}+c_{i, j}(s)^{-\delta}+c_{i, j}(t)^{-\delta}\right) \\
& \leqslant 2 M \sum_{1 \leqslant i, j \leqslant \ell} \sum_{m<s \leqslant n} \sum_{m<t \leqslant n}\left(b_{i, j}(s, t)^{-\delta}+c_{i, j}(t)^{-\delta}\right) .
\end{aligned}
$$

By writing $c_{i, j}(t)$ increasingly for $m<t \leqslant n$ and using the equivalent description of (2.7) before the proof, we have

$$
\sum_{m<t \leqslant n} c_{i, j}(t)^{-\delta} \leqslant 2^{\delta} \sum_{t=1}^{n-m} M^{\delta} t^{-\delta}
$$

Suppose (2.8) holds for $b_{i, j}$, then similar estimate using (2.8) gives

$$
\sum_{m<t \leqslant n} b_{i, j}(s, t)^{-\delta} \leqslant 2^{\delta} \sum_{t=1}^{n-m} M^{\delta} t^{-\delta} .
$$


By (2.13) and (2.14), we have

$$
\begin{aligned}
\sum_{m<t \leqslant n}\left(b_{i, j}(s, t)^{-\delta}+c_{i, j}(t)^{-\delta}\right) & \leqslant 2^{1+\delta} \sum_{t=1}^{n-m} M^{\delta} t^{-\delta} \leqslant 2^{2+\delta} M^{\delta} \int_{1 / 2}^{n-m} t^{-\delta} \mathrm{d} t \\
& \leqslant\left\{\begin{array}{lr}
2^{2+\delta} M^{\delta}(1-\delta)^{-1}(n-m)^{1-\delta} & \text { if } 0<\delta<1 \\
2^{2+\delta} M \log 2(n-m) & \text { if } \delta=1 . \\
2^{1+2 \delta} M^{\delta}(\delta-1)^{-1} & \text { if } \delta>1
\end{array}\right.
\end{aligned}
$$

If (2.9) holds for $b_{i, j}$, then one obtains similar estimate to (2.15). We assume without loss of generality that (2.8) holds for all $b_{i, j}$.

Using (2.15) and (2.12) in different cases according to $\delta$, we will have (2.1) for various $\sigma$. If $\delta>1$, then

$$
E(m, n) \leqslant 4^{1+\delta} M^{1+\delta}(\delta-1)^{-1} \ell^{2}(n-m) .
$$

So (2.1) holds for $\sigma=1$ and the conclusion in this case follows from Theorem 2.1 .

If $\delta=1$, then

$$
E(m, n) \leqslant 2^{3+\delta} M^{2} \ell^{2}(n-m) \log 2(n-m) \ll(n-m)^{1+\varepsilon},
$$

where the implied constant depends on $M, \ell$ and $\varepsilon$. We claim that for any nonnegative integers $m<n$,

$$
(n-m)^{1+\varepsilon} \ll n^{1+\varepsilon}-m^{1+\varepsilon},
$$

where the implied constant may depend on $\varepsilon$ but not on $m$ or $n$. We prove the claim by considering two cases. If $m \leqslant \frac{n}{2}$, then by the mean value theorem, there exists $x \in[0,1 / 2]$ such that

$$
\begin{aligned}
(n-m)^{1+\varepsilon} & =n^{1+\varepsilon}(1-(m / n))^{1+\varepsilon}=n^{1+\varepsilon}\left(1-(1+\varepsilon)(1-x)^{\varepsilon} m / n\right) \\
& \leqslant n^{1+\varepsilon}-(1+\varepsilon) 2^{-\varepsilon} m n^{\varepsilon} \leqslant n^{1+\varepsilon}-m^{1+\varepsilon} .
\end{aligned}
$$

If $n / 2<m<n$, then

$$
(n-m)^{1+\varepsilon}=(n-m)(n-m)^{\varepsilon} \leqslant(n-m)(n / 2)^{\varepsilon} \leqslant 2^{-\varepsilon}\left(n^{1+\varepsilon}-m^{1+\varepsilon}\right) .
$$

This completes the proof of the claim. By (2.16) and (2.17), we have (2.1) holds for $\sigma=1+\varepsilon$ and the conclusion in this case follows from Theorem 2.1.

If $0<\delta<1$, then by (2.12), (2.15) and (2.17),

$$
E(m, n) \leqslant 2^{3+\delta} M^{1+\delta}(1-\delta)^{-1} \ell^{2}(n-m)^{2-\delta} \ll n^{2-\delta}-m^{2-\delta},
$$

where the implied constant is independent of $m$ or $n$. So (2.1) holds for $\sigma=2-\delta$ and the conclusion in this case follows from Theorem 2.1. 


\section{EFFECTIVE DECAY OF CORRELATIONS}

The aim of this section is to formulate a simplified version of Theorem 2.2 under the assumption of effective decay of correlations. This simplified version can be applied directly to prove Theorem 1.3. After that we prove Theorem 1.8. At the end of this section we prove Example 1.7.

To make our statement applicable to broad contexts and explain the assumption (2.6), we consider a class of measurable transformations $\mathcal{H}$ on a probability space $(X, \mathcal{X}, \mu)$. We remark here that we don't assume elements of $\mathcal{H}$ preserve $\mu$.

For $k \geqslant 1$, we use dist $\left(t_{0}, t_{1}, \ldots, t_{k}\right)$ to denote the minimal distance between $k+1$ real numbers, i.e.,

$$
\operatorname{dist}\left(t_{0}, t_{1}, \ldots, t_{k}\right)=\min _{i \neq j}\left|t_{i}-t_{j}\right|
$$

We say the action of $\mathcal{H}$ on $(X, \mu)$ has $k$-multiple $n^{-\delta}$ decay of correlations for a vector space of measurable functions $\mathcal{F}_{0}$ if the following holds: there exists an injective map $t: \mathcal{H} \rightarrow \mathbb{R} \backslash\{0\}$ so that for any $h_{1}, \ldots, h_{k} \in \mathcal{H}$, any $f_{1}, \ldots, f_{k} \in \mathcal{F}_{0}$ and any $r_{1}, \ldots, r_{k} \in \mathbb{N} \cup\{0\}$, one has

$$
\left|\int_{X} f_{1}\left(h_{1}^{r_{1}} x\right) \cdots f_{k}\left(h_{k}^{r_{k}} x\right) \mathrm{d} \mu(x)\right| \ll\left(\operatorname{dist}\left(0, t\left(h_{1}\right) r_{1}, \ldots, t\left(h_{k}\right) r_{k}\right)+1\right)^{-\delta}
$$

where the implied constant may depend on $f_{1}, \ldots, f_{k}, h_{1}, \ldots, h_{k}$ but is independent of $r_{1}, \ldots, r_{k}$. Although in this paper we only consider invariant measures, the formulation of effective decay of correlations in (3.1) is natural when $\mu$ is not invariant by the action, see [22]. Another aim of this general setting is to explain the assumption (2.6) in Theorem 2.2.

Theorem 3.1. Suppose the action of $\mathcal{H}$ on $(X, \mu)$ has $2 \ell$-multiple $n^{-\delta}(\delta>0)$ decay of correlations for a vector space of measurable functions $\mathcal{F}_{0}$. Then for any $\varepsilon>0$, any pairwise distinct maps $h_{1}, \ldots, h_{\ell} \in \mathcal{H}$, any $f_{1}, \ldots, f_{\ell} \in \mathcal{F}_{0}$ and any non-clustered sequence of positive integers $\left\{r_{n}\right\}$, one has $\mu$-almost surely

$$
\frac{1}{N} \sum_{n=1}^{N} f_{1}\left(h_{1}^{r_{n}} x\right) \cdots f_{\ell}\left(h_{\ell}^{r_{n}} x\right)=o_{x}\left(\rho_{\varepsilon, \delta}(N)\right),
$$

where $\rho_{\varepsilon, \delta}(N)$ is as in Theorem 1.3 .

Proof. To put the left-hand side of (3.2) into the framework of Theorem 2.2 we take $Y=X, \xi_{i}=\mathrm{Id}, \nu=\mu$ and $h_{i, n}=h_{i}^{r_{n}}$, where $1 \leqslant i \leqslant \ell$ and $n \in \mathbb{N}$. Let $t: \mathcal{H} \rightarrow \mathbb{R} \backslash\{0\}$ be the injective map given by the definition of $2 \ell$-multiple $n^{-\delta}$ decay of correlations, i.e., (3.1) holds for $k=2 \ell$. Then $t_{i}=t\left(h_{i}\right)(1 \leqslant i \leqslant \ell)$ are pairwise distinct and nonzero real numbers. Recall that $\left\{r_{n}\right\}$ is a nonclustered sequence of positive integers. 
For $1 \leqslant i, j \leqslant \ell$ and $m, n \in \mathbb{N}$, we take

$$
\begin{aligned}
b_{i, j}(m, n) & =\left|t_{i} r_{m}-t_{j} r_{n}\right|+1, \\
c_{i, j}(n) & =\left\{\begin{array}{ll}
\left|t_{i} r_{n}-t_{j} r_{n}\right|+1 & \text { if } i \neq j \\
\left|t_{i} r_{n}\right|+1 & \text { if } i=j
\end{array} .\right.
\end{aligned}
$$

It follows from (3.1) with $k=2 \ell$ that (2.6) holds with $\delta$ as in the statement and some $M \geqslant 1$ independent of $m$ or $n$. We show that for any $i, j \in\{1,2, \ldots, \ell\}$ one has that (2.10) and (2.11) hold, which implies (2.7) and (2.8). In view of Theorem [2.2, this will complete the proof.

For (2.11), it suffices to prove that the cardinality of $n \in \mathbb{N}$ satisfying

$$
t_{j} r_{n}-t_{i} r_{m} \in[s, s+1] \Leftrightarrow r_{n} \in t_{j}^{-1}\left[s+t_{i} r_{m}, s+1+t_{i} r_{m}\right]
$$

is uniformly bounded for all $s \in \mathbb{Z}$ and $m \in \mathbb{N}$. Note that the interval $t_{j}^{-1}[s+$ $t_{i} r_{m}, s+1+t_{i} r_{m}$ ] has length $t_{j}^{-1}$ which is independent of $m$ or $s$. Since we assume the sequence $\left\{r_{n}\right\}$ is non-clustered, there exists $M \geqslant 1$ such that

$$
\left|\left\{n \in \mathbb{N}: r_{n} \in I\right\}\right| \leqslant M
$$

for any closed interval $I$ whose length is no more than $t_{j}^{-1}$. Therefore (2.11) holds. For $i \neq j$, one can prove (2.10) similarly using $t_{i} \neq t_{j}$. The same argument using $t_{i} \neq 0$ proves (2.10) in the case where $i=j$.

Proof of Theorem 1.3. Let $\mathcal{F}_{0}=\{f \in \mathcal{F}: \mu(f)=0\}$, which is a subspace of $\mathcal{F}$ since $\mathcal{F}$ contains constant functions. We assume that $\mathcal{F}_{0} \neq\{0\}$, otherwise the conclusion holds trivially. This assumption and (1.4) imply $h^{m} \neq h^{n}$ for $m \neq n$. We take $\mathcal{H}=\left\{h^{m}: m \in \mathbb{N}\right\}$ and $t\left(h^{m}\right)=m$. For any $k \in \mathbb{N}$, if (1.4) holds for $f_{i} \in \mathcal{F}$, then (3.1) holds for $f_{i} \in \mathcal{F}_{0}$. Since we assume $\mathcal{F}$ contains constant functions, the $(2 \ell-1)$-mixing assumption implies (1.4) and hence (3.1) for any $1 \leqslant k \leqslant 2 \ell-1$. So Theorem 3.1 can be applied in this setting with $\ell$ replaced by any element of $\{1, \ldots, \ell\}$. A simple induction argument on $\ell$ shows that equation (1.5) holds for any $f_{i} \in \mathcal{F}$. The proof of the Remark 1.5 is the same with $\mathcal{H}=\left\{h^{m}: m \in \mathbb{Z}, m \neq 0\right\}$.

Proof of Theorem 1.8. Since $\mathcal{L} \cap L^{\infty}(X, \mu)$ contains constant functions, by induction on $k$, it suffices to show that for any $k \in \mathbb{N}$, any $f_{0}, \ldots, f_{k} \in \mathcal{L} \cap$ $L^{\infty}(X, \mu)$ with $\mu\left(f_{0}\right)=0$ and any pairwise distinct natural numbers $n_{1}, \ldots, n_{k}$, we have

$$
\left|\int_{X} f_{0}(x) f_{1}\left(h^{n_{1}} x\right) \cdots f_{k}\left(h^{n_{k}} x\right) \mathrm{d} \mu(x)\right| \ll\left(\min \left\{n_{1}, \ldots, n_{k}\right\}\right)^{-\delta}
$$

where the implied constant depends on $f_{0}, \ldots, f_{k}$. To see this, we assume without loss of generality that $n_{1}<n_{2}<\cdots<n_{k}$ and apply (1.6) for $\psi=f_{0}$, 
$\varphi=f_{1}(x) f_{2}\left(h^{n_{2}-n_{1}} x\right) \cdots f_{k}\left(h^{n_{k}-n_{1}} x\right)$ and $n=n_{1}$. Then we have the left hand side of (3.5) is

$$
\leqslant C\left\|f_{1}\right\|_{L^{\infty}} \cdots\left\|f_{k}\right\|_{L^{\infty}}\left\|f_{0}\right\|_{\mathcal{L}} n_{1}^{-\delta}
$$

which implies (3.5).

Proof of Example 1.7. The effective multiple mixing was proved in [18] with a different language. Let $k \in \mathbb{N}$ and $\mathbf{f}=\left(f_{0}, f_{1}, \ldots, f_{k}\right)$ where $f_{i} \in C^{\theta}(X)$. [18] considers

$$
\operatorname{Cov}(\mathbf{f}):=\left.\frac{\partial^{k+1}}{\partial \theta_{0} \cdots \partial \theta_{k}} \log \left(\int_{X} \exp \left(\sum_{i=0}^{k} \theta_{i} f_{i}\right) \mathrm{d} \mu\right)\right|_{\theta_{0}=\cdots=\theta_{k}=0} .
$$

It follows from [18, Proposition 3.1] that there exists $C, \sigma>0$ such that for any integers $n_{1}, \ldots, n_{k}$

$$
\left|\operatorname{Cov}\left(f_{0}, f_{1} \circ h^{n_{1}}, \ldots, f_{k} \circ h^{n_{k}}\right)\right| \leqslant C e^{-\sigma\left(\max _{i}\left|n_{i}\right|\right)} .
$$

Here $C$ may depend on $f_{0}, \ldots, f_{k}$, but we may choose one which works for a fixed finite family of functions and bounded $k$. The independence of $\sigma$ from $f_{i}$ is not explicitly stated in [18, Proposition 3.1], but it can be deduced from the proof.

For a nonempty subset $I=\left\{i_{0}<\cdots<i_{s}\right\}$ of $\{0, \ldots, k\}$, we take

$$
\mathbf{f}_{I}=\left(f_{i_{0}} \circ h^{n_{i_{0}}}, \ldots, f_{i_{s}} \circ h^{n_{i_{s}}}\right) .
$$

By a partition $P=\left\{I_{1}, \ldots, I_{s}\right\}$ of $\{0, \ldots, k\}$, we mean a decomposition of $\{0, \ldots, k\}$ into a disjoint union of elements of $P$. We use $\mathcal{P}(k)$ to denote the set of partitions of $\{0, \ldots, k\}$. By [18, Lemma 2.1],

$$
\int_{X} f_{0} \circ h^{n_{0}} f_{1} \circ h^{n_{1}} \cdots f_{k} \circ h^{n_{k}} \mathrm{~d} \mu=\sum_{P \in \mathcal{P}(k)} \prod_{I \in P} \operatorname{Cov}\left(\mathbf{f}_{I}\right) .
$$

If $I=\left\{i_{0}<i_{1}<\cdots<i_{n}\right\}$ where $n \geqslant 1$, then the $h$-invariance of $\mu$ implies

$$
\operatorname{Cov}\left(\mathbf{f}_{I}\right)=\operatorname{Cov}\left(f_{i_{0}}, f_{i_{1}} \circ h^{n_{i_{1}}-n_{i_{0}}}, \cdots, f_{i_{s}} \circ h^{n_{i_{s}}-n_{i_{0}}}\right) .
$$

This together with (3.6) implies

$$
\left|\operatorname{Cov}\left(\mathbf{f}_{I}\right)\right| \leqslant C \sum_{t=1}^{s} e^{-\sigma\left|n_{i_{t}}-n_{i_{0}}\right|} .
$$

Note that the continuous functions $f_{i}$ are bounded on the compact space $X$. So if $P$ contains an $I$ with $|I|>1$, in view of (3.8) we have

$$
\prod_{I \in P}\left|\operatorname{Cov}\left(\mathbf{f}_{I}\right)\right| \ll \sum_{t=1}^{s} e^{-\sigma\left|n_{i_{t}}-n_{i_{0}}\right|} .
$$


For the $P$ consists of only singletons, by the $h$-invariance of $\mu$, we have

$$
\prod_{I \in P} \operatorname{Cov}\left(\mathbf{f}_{I}\right)=\prod_{i=0}^{k} \int_{X} f_{i} \mathrm{~d} \mu
$$

Therefore the system $(X, \mu, h)$ is $k$-mixing with exponential rate for $C^{\theta}(X)$.

\section{SUBGROUP ACTIONS ON HOMOGENEOUS SPACES}

The aim of this section is to prove Theorem 1.1. Let the notation and assumptions be as in Theorem 1.1. Recall that $H$ is a semi-simple Lie group with finite center. We fix a maximal compact subgroup $K$ of $H$ and denote by Ad to be the adjoint representation. We endow the Lie algebra $\mathfrak{h}$ of $H$ with an $\operatorname{Ad}(K)$ invariant inner product. This inner product induces a right invariant Riemannian metric $\rho$ on $H$ and a Hermitian inner product on $\mathfrak{h}_{\mathbb{C}}=\mathfrak{h} \otimes \mathbb{C}$. For any $h \in H$, let $\|\operatorname{Ad}(h)\|$ be the operator norm on $\mathfrak{h}$ and $\mathfrak{h}_{\mathbb{C}}$, that is,

$$
\|\operatorname{Ad}(h)\|=\max \{\|\operatorname{Ad}(h) v\|: v \in \mathfrak{h},\|v\|=1\}=\max \left\{\|\operatorname{Ad}(h) v\|: v \in \mathfrak{h}_{\mathbb{C}},\|v\|=1\right\} .
$$

By [2, Lemma 2.1(iii)], there exists $0<\kappa \leqslant 1 \leqslant \tau$ such that for any $h \in H$

$$
\|\operatorname{Ad}(h)\|^{\kappa} \ll \exp \rho\left(h, 1_{H}\right) \ll\|\operatorname{Ad}(h)\|^{\tau} .
$$

Proof of Theorem 1.1. It suffices to show that (1.2) holds for $\mu$-almost every $x$ under the additional assumption that that $\mu\left(f_{i}\right)=0$ and $f_{i}=\tilde{f}_{i}+c_{i}$ where $\tilde{f}_{i} \in C_{c}^{\infty}(X)$ and $c_{i} \in \mathbb{R}$. We will apply Theorem 2.2 to

$$
F_{n}(x)=\prod_{i=1}^{\ell} f_{i}\left(h_{i}^{r_{n}} x\right)
$$

that is, in the case where $X=Y, \xi_{i}=\operatorname{Id}$ and $h_{i, n}=h_{i}^{r_{n}}$. We take

$$
\begin{aligned}
b_{i, j}(m, n) & =\left\|\operatorname{Ad}\left(h_{i}^{m} h_{j}^{-n}\right)\right\|, \\
c_{i, j}(n) & =\left\{\begin{array}{cl}
\left\|\operatorname{Ad}\left(h_{i}^{n} h_{j}^{-n}\right)\right\| & \text { if } i \neq j \\
\infty & \text { if } i=j
\end{array} .\right.
\end{aligned}
$$

The existence of $M, \delta>0$ so that (2.6) holds follows from [2, Theorem 1.1] and (4.1).

Now we show that (2.7) holds for any $c_{i, j}$ with $i \neq j$. The group generated by $h=\operatorname{Ad}\left(h_{i} h_{j}^{-1}\right)$ is unbounded by the assumption. If $h$ is not quasi-unipotent, then there is an eigenvalue $r \in \mathbb{C}$ with $|r|>1$ for the action of $\operatorname{Ad}(h)$ on $\mathfrak{h}_{\mathbb{C}}$. So $c_{i, j}(n) \geqslant|r|^{n} \gg n$, which implies (2.7). If $h$ is quasi-unipotent and generates an unbounded subgroup, then in view of the Jordan canonical form 
of $h$, there exists a two dimensional $h$-invariant subspace $V$ of $\mathfrak{h}_{\mathbb{C}}$ such that with respect to some basis of $V$

$$
\left.h\right|_{V}=\left(\begin{array}{cc}
s & 1 \\
0 & s
\end{array}\right) \quad \text { and }\left.\quad h^{n}\right|_{V}=\left(\begin{array}{cc}
s^{n} & n s^{n-1} \\
0 & s^{n}
\end{array}\right)
$$

where $|s|=1$. Therefore,

$$
\left\|h^{n}\right\| \geqslant\left\|\left.h^{n}\right|_{V}\right\| \gg n
$$

where the implied constant depends on the choice of the basis. Therefore (2.7) holds.

Next, we show that either (2.8) or (2.9) holds for each $b_{i, j}$. Let $h=\operatorname{Ad}\left(h_{i}\right)$ and $g=\operatorname{Ad}\left(h_{j}^{-1}\right)$. The groups $\langle h\rangle$ and $\langle g\rangle$ are unbounded according to the assumption. We consider two cases.

First, suppose both $h$ and $g$ are quasi-unipotent. We have the Jordan decomposition $g=g_{s} g_{u}=g_{u} g_{s}$ where $g_{s} \in \mathrm{GL}(\mathfrak{h})$ is semi-simple and $g_{u} \in \mathrm{GL}(\mathfrak{h})$ is unipotent, see [3, §I.4.4]. Since $g$ is quasi-unipotent and $\langle g\rangle$ is unbounded, we have $\left\langle g_{s}\right\rangle$ is relatively compact and $g_{u}$ is non-trivial. Similarly, we have the Jordan decomposition $h=h_{s} h_{u}$. Moreover, since $g$ and $h$ commute with each other, all the elements appearing in the Jordan decomposition commute with each other. Therefore, there exists $C \geqslant 1$ such that

$$
C^{-1}\left\|h_{u}^{m} g_{u}^{n}\right\| \leqslant\left\|h^{m} g^{n}\right\| \leqslant C\left\|h_{u}^{m} g_{u}^{n}\right\| .
$$

So we can assume without loss of generality that $g$ and $h$ are unipotent. By Engel's theorem, there is a basis of $\mathfrak{h}$ such that $g$ and $h$ are upper triangular unipotent matrices. There exists a non-diagonal entry $s$ of $g$ such that $s \neq 0$ and all the other non-diagonal entries of $g$ left to $s$ are zero, i.e. one row of $g$ has the form

$$
\left(\begin{array}{lllllllll}
0 & \cdots & 0 & 1 & 0 & \cdots & 0 & s & \cdots
\end{array}\right) .
$$

The same row of $h^{m} g^{n}$ is

$$
\left(\begin{array}{lllllllll}
0 & \cdots & 0 & 1 & 0 & \cdots & 0 & s n+t_{m} & \cdots
\end{array}\right)
$$

where $t_{m}$ is the entry of $h^{m}$ at the same position of $s$. It follows that $\left\|h^{m} g^{n}\right\| \gg$ $\left|s n+t_{m}\right|$, which implies (2.8) with $M$ comparable to $s^{-1}$ but most importantly independent of $m$.

In the second case we assume at least one of $g$ and $h$ is not quasi-unipotent. Suppose there exists a simultaneous eigenvector $v \in \mathfrak{h}_{\mathbb{C}}$ of $g$ and $h$, say

$$
g v=s v \quad \text { and } \quad h v=t v,
$$

such that $\max \{|s|,|t|\}>1$ and $\min \{|s|,|t|\} \geqslant 1$. Then either $\left\|h^{m} g^{n}\right\| \geqslant$ $(\max \{|s|,|t|\})^{m}$ or $\left\|h^{m} g^{n}\right\| \geqslant(\max \{|s|,|t|\})^{n}$, which implies that either (2.8) 
or (2.9) holds. Note that we can always find such an eigenvector $v$ if precisely one of $g$ and $h$ is quasi-unipotent: $v$ is taken to be a simultaneous eigenvector associated to an eigenvalue of modulus strictly greater than one.

Suppose that none of the simultaneous eigenvectors have the property considered in the previous paragraph, then both of $g$ and $h$ are not quasiunipotent. Moreover, every simultaneous eigenvector expanded by $g$ is contracted by $h$ (and vice versa). Let $S_{+}$(resp. $S_{-}$) denote the set of eigenvalues of $g$ with modulus $>1$ (resp. $<1$ ) appearing with multiplicity. Similarly $T_{ \pm}$denote the corresponding eigenvalues for $h$. By assumption we have a bijection between $S_{ \pm}$and $T_{\mp}$, and we denote either map by $s \mapsto t_{s}$.

Fix $m \in \mathbb{N}$. Let $k \in \mathbb{N}$ be the maximal positive integer such that $\left|s^{n} t_{s}^{m}\right|<1$ for all $n<k$ and all $s \in S_{+}$. Let $s_{+} \in S_{+}$for which $\left|s_{+}^{k} t_{s_{+}}^{m}\right|>1$. Since $g, h \in \mathrm{SL}(\mathfrak{h})$, there exists an eigenvalue $s_{-} \in S_{-}$such that $\left|s_{-}^{(k-1)} t_{s_{-}}^{m}\right|>1$. Therefore,

$$
\left\|h^{m} g^{n}\right\| \geqslant \frac{1}{2}\left(\left|s_{+}^{n} t_{s_{+}}^{m}\right|+\left|s_{-}^{n} t_{s_{-}}^{m}\right|\right) \geqslant \frac{1}{2} \min \left\{\left|s^{+}\right|,\left|t_{s^{-}}\right|\right\}^{|n-k|-1} .
$$

Note that the lower bound above is an exponential function whose base is bigger than one and independent of $m$, so (2.8) holds.

If all the $h_{i}$ and $h_{i} h_{j}^{-1}(i \neq j)$ are not quasi-unipotent, then $c_{i, j}(n)(i \neq j)$ and $b_{i, j}(m, n)$ are bounded from below by exponential functions whose base and coefficients are independent of $m$. In this case the bound of (2.6) is the same as that in exponential decay of correlations, so we have a better error term (1.3).

\section{REFERENCES}

[1] I. Assani, Multiple recurrence and almost sure convergence for weakly mixing dynamical systems, Israel J. Math. 103 (1998), 111-124.

[2] M. Björklund, M. Einsiedler and A. Gorodnik, Quantitative multiple mixing, to appear in Journal of the Eur. Math. Soc. (2017).

[3] A. Borel, Linear algebraic groups. Second edition, Graduate Texts in Mathematics, 126. Springer-Verlag, New York, 1991.

[4] J. Bourgain, On the maximal ergodic theorem for certain subsets of the integers, Israel J. Math. 61 (1988), no. 1, 39-72.

[5] J. Bourgain, Pointwise ergodic theorems for arithmetic sets, With an appendix by the author, H Furstenberg, Y. Katznelson and Donald S. Ornstein, Inst. Hautes Études Sci. Publ. Math. No. 69 (1989), 5-45.

[6] J. Bourgain, Double recurrence and almost sure convergence, J. Reine Angew. Math. 404 (1990), 140-161.

[7] R. Bowen, Equilibrium States and the ergodic theory of Anosov diffeomorphisms, Second revised edition, With a preface by David Ruelle, Edited by Jean-René Chazottes. Lecture Notes in Mathematics, 470. Springer-Verlag, Berlin, 2008. 
[8] J. Derrien and E. Lesigne, Un théorème ergodique polynomial ponctuel pour les endomorphismes exacts et les K-systèmes, (French) [A pointwise polynomial ergodic theorem for exact endomorphisms and K-systems], Ann. Inst. H. Poincar Probab. Statist., 32 (1996), 765-778.

[9] D. Dolgopyat, Limit theorems for partially hyperbolic systems, Trans. Amer. Math. Soc. 356 (2004), 1637-1689.

[10] S. Donoso and W. Sun, Pointwise multiple averages for systems with two commuting transformations, Ergodic Theory Dynam. Systems 38 (2018), no. 6, 2132-2157.

[11] S. Donoso and W. Sun, Pointwise convergence of some multiple ergodic averages, Adv. Math. 330 (2018), 946-996.

[12] H. Furstenberg, Recurrence in ergodic theory and combinatorial number theory, M. B. Porter Lectures, Princeton University Press, Princeton, N.J., 1981.

[13] E. Glasner, Ergodic theory via joinings, Mathematical Surveys and Monographs 101, American Mathematical Society, Providence, RI, 2003.

[14] A. Gorodnik and R. Spatzier, Exponential mixing of nilmanifold automorphisms, J. Anal. Math. 123 (2014), 355-396.

[15] Y. Gutman, W. Huang, S. Shao and X. Ye, Almost sure convergence of the multiple ergodic average for certain weakly mixing systems, Acta Math. Sin. (Engl. Ser.) 34 (2018), no. 1, 79-90.

[16] W. Huang, S. Shao and X. Ye, Pointwise convergence of multiple ergodic averages and strictly ergodic models, J. Analyse Math., to appear

[17] D. Kleinbock, R. Shi and B. Weiss, Pointwise equidistribution with an error rate and with respect to unbounded functions, Math. Ann. 367 (2017), 857-879.

[18] M. Kotani and T. Sunada, The pressure and higher correlations for an Anosov diffeomorphism, Ergodic Theory Dynam. Systems 21 (2001), no. 3, 807-821.

[19] P. Sarnak and A. Ubis, The horocycle flow at prime times, J. Math. Pures Appl. (9) 103 (2015), no. 2, 575-618.

[20] W. Schmidt, A metrical theorem in diophantine approximation, Canad. J. Math. 12 1960, 619-631.

[21] N.A. Shah, Limit distributions of polynomial trajectories on homogeneous spaces, Duke Math. J. 75 (1994), no. 3, 711-732.

[22] R. Shi, Expanding cone and applications to homogeneous dynamics, With an appendix by R. Rühr and the author, to appear in IMRN.

[23] V.G. Sprindzhuk, Metric theory of Diophantine approximations, Halsted Press, 1979.

[24] T. Tao, Norm convergence of multiple ergodic averages for commuting transformations, Ergod. Th. and Dynam. Sys. 28 (2008), 657-688.

[25] P. Walters, An introduction to ergodic theory, Graduate Texts in Mathematics, 79. Springer-Verlag, New York-Berlin, 1982.

[26] M. Walsh, Norm convergence of nilpotent ergodic averages, Ann. of Math. (2) 175 (2012), 1667-1688.

[27] L.-S. Young, Recurrence times and rates of mixing, Israel J. Math. 110 (1999), 153-188.

Faculty of Mathematics, Technion, Haifa, IsRael

E-mail address: rener@campus.technion.ac.il

Shanghai Center for Mathematical Sciences, Jiangwan Campus, Fudan UniVersity, No.2005 Songhu Road, Shanghai, 200433, China

E-mail address: ronggang@fudan.edu.cn 